\title{
Controversies in the management of the renal artery stenosis
}

\author{
Khalil Kanjwal ${ }^{1}$, Vincent M. Figueredo ${ }^{2}$ \\ ${ }^{1}$ Clinical Cardiac Elelctrophysiology Fellow, Johns Hopkins University, Baltimore, MD, USA \\ ${ }^{2}$ Heart and Vascular Institute, Einstein Medical Center, Philadelphia, PA, USA
}

\begin{abstract}
Optimal management of renal artery stenosis has continued to remain elusive. The previous non randomized studies and registry data suggested a benefit of renal artery stenting. However, the recently completed randomized studies comparing renal stenting to medical management failed to show any benefit. These studies had some flaws in their design and methodology. In an appropriately selected patient population renal artery stenting may have a role. In addition, there might be some role of adjunctive therapies like antiplatelet medications and embolic protection. This review summarizes the current literature on this controversial topic. (Cardiol J 2013; 20, 1: 11-16)
\end{abstract}

Key words: kidney, renal artery stenosis, stenting

\section{Introduction}

Renal artery stenosis (RAS) affects approximately $5 \%$ of the 50 million people with hypertension in Unites States. In patients older than 50 years RAS may account for up to $15 \%$ of chronic renal failure and $20 \%$ with end stage renal disease. The prevalence of RAS of greater than $60 \%$ luminal narrowing is approximately $7 \%$ in patients who are 65 years of age or older [1]. Most cases of RAS result from arteriosclerosis of the renal artery. In minority, especially in younger subjects it may result from fibromuscular dysplasia. In one study of the Medicare population, the prevalence of symptomatic atherosclerotic RAS was $0.5 \%$ overall and approximately $5.5 \%$ among those with chronic kidney disease [2]. In another study of elderly population involving duplex ultrasonography, the prevalence was $7 \%$ [3]. The prevalence of the atherosclerotic RAS increases with age, particularly in patients who suffer from diabetes, hyperlipidemia, peripheral vascular disease, coronary disease and hypertension.
Caps et al. [4] found that $50 \%$ of patients with RAS greater than $60 \%$ progressed at the follow-up of 33 months. However, only $3 \%$ progressed to complete occlusion in this study. There is a close association between severity of RAS and renal atrophy leading to ischemic nephropathy $[4,5]$.

\section{Fibromuscular dysplasia}

Fibromuscular dysplasia accounts for less than $10 \%$ of all cases of RAS. It is usually seen in young ( $<40$ years) females and involves the mid or distal segments of the renal artery. Fibromuscular dysplasia can be successfully treated with angioplasty. In a study of Tegtmeyer et al. [6] percutaneous transluminal renal angioplasty (PTRA) was used to treat 66 patients with 85 renal artery stenoses due to fibromuscular dysplasia. The initial success rate for the procedure was $100 \%$. The recurrence rates were $8 \%$ of lesions and $10 \%$ of patients. Cumulative patency rate predicted for 10 years was $87.07 \%$. The mean systolic pressure decreased by $52 \mathrm{~mm}$

Address for correspondence: Khalil Kanjwal, MD, Fellow Cardiovascular Medicine, Johns Hopkins University, 1800 Orleans street, 21287 Baltimore, MD, USA, e-mail: Khalilkanjwal@yahoo.com

Received: 07.08.2012

Accepted: 10.10.2012 
$\mathrm{Hg}$ and the mean diastolic pressure decreased by $35 \mathrm{~mm} \mathrm{Hg}$ in response to treatment. Approximately, $40 \%$ of the patients were cured, $60 \%$ were classified as improved, and only $1(2 \%)$ did not respond to PTRA. Renal function was improved in $86 \%$ of the patients and stabilized in $14 \%$ of the patients.

The optimal medical management for the atherosclerotic RAS (ARAS) continues to remain controversial. Refractory hypertension and end stage renal disease may occur in patients with advanced RAS but the incidence of these outcomes was reported to be low in patients who are treated medically $[4,7,8]$. Except for patients with renal artery occlusion there seems to be a poor correlation between severity of the stenosis and the renal function $[9,10]$.

\section{Atherosclerotic renal artery stenosis and cardiovascular outcomes}

Patients suffering from ARAS are at a higher risk of cardiovascular events. In one study the rate of chronic kidney disease was $25 \%$ vs. $2 \%$ in patients with and without ARAS. Similarly, the incidence of coronary artery disease was $67 \%$ vs. $25 \%$, stroke was $37 \%$ vs. $12 \%$ and that of peripheral vascular disease was $56 \%$ vs. $13 \%$, respectively [2]. Renal insufficiency in patients with ARAS has been associated with decreased survival [11]. The long-term cardiovascular outcomes following coronary angiographies were significantly higher in patients with concomitant ARAS than those without $[12,13]$. ARAS has also been associated with left ventricular dysfunction and congestive heart failure [14, 15]. Conversely, improvement in renal function in patients with ARAS following PTRA has been reported to improve survival as well as quality of life [16-18].

\section{Current evidence on the role of PTRA and stenting in patients with ARAS}

As has been previously alluded to, the role of renal angioplasty and stenting has remained controversial. Leertouwer et al. [19] performed metaanalysis on the studies of renal arterial stent placement (14 articles, 678 patients) and renal PTA (10 articles, 644 patients) published up to August 1998. A random-effects model was used to pool the data. The mean follow-up was approximately 17-19 months. In the PTRA alone arm of this study technical success was achieved in $77 \%$ of patients. Hypertension was cured in $10 \%$ and improved in 53\% of the patients. Renal insufficiency stabilized in $41 \%$ and improved in $38 \%$ patients. In the renal stent arm of the meta-analysis technical success rate was $98 \%$. Hypertension improved in $49 \%$ and was cured in $20 \%$. Renal insufficiency improved in $30 \%$ and stabilized in $38 \%$ of these patients. The restenosis (> 50\% at 6 months) was $26 \%$ in PTRA alone group vs. $17 \%$ in the renal artery stenting group. Interestingly the cure rate for hypertension was higher and the improvement rate for renal function was lower after stent placement than after renal PTA (20\% vs. $10 \%$ and $30 \%$ vs. $38 \%$, respectively; $p<0.001$ ).

\section{Stenting vs. medical management}

Three small randomized, controlled trials showed no benefit of renal stenting combined with medical management over medical management alone [20-22]. A significant improvement in blood pressure (BP) however was noted in the renal stenting arm of one of the studies including patients with bilateral stenosis [21]. However, these earlier trials was that they were small sized and thus underpowered to detect any clinically significant benefit. These trials evaluated surrogate end points like BP, creatinine and reduction in number of hypertensive medications. The medical regimens designed were inadequate and inconsistent. They enrolled patients with $<50 \%$ stenosis and the crossover between the treatment arms was allowed but analysis was done on the basis of intention to treat. There were no standardized core laboratories for the assessment of renal angiograms. The biggest flaw of these small trials was that they lacked the analysis on cardiovascular outcomes.

\section{Recent randomized trials comparing stenting plus medical therapy with medical therapy alone}

Three recently published randomized controlled trials no benefit of medical therapy with stenting when compared to medical therapy alone [23-25].

\section{NITER trial}

Nephropathy Ischemic Therapy trial [23] included the 52 elderly patients with $>70 \%$ stenosis. The diagnosis in each of these patients was obtained by Doppler and confirmed by magnetic resonance angiography (MRA). Endpoints of this study included death, initiation on dialysis, decline in estimated glomerular filtration rate (eGFR) by $20 \%$ and hospitalization. There was no benefit of preserved renal function or improved survival observed when stenting was added to medical management. 
However, there were some serious limitations in the design of this study. This was a small study in which the diagnosis of RAS was primarily made with the Doppler/MRA. The medical therapy was not precisely defined. There was crossover allowed in the study and the study lacked a core laboratory adjudication.

\section{STAR trial}

Stenting in Renal Dysfunction Caused by Atherosclerotic Renal Artery (STAR) trial [24] was a multi centric, randomized clinical trial conducted at 10 European centers including, 140 patients with eGFR $<80 \mathrm{~mL} / \mathrm{min} / 1.73 \mathrm{~m}^{2}$ and a renal stenosis of greater than $50 \%$. The primary endpoint was greater than a $20 \%$ decline in eGFR. Seventy six patients were randomized to medical treatment only and 64 patients to both medical treatment and stenting. Of the 64 patients randomized to the stent only 46 patients actually received the stent. Sixteen percent of patients in medical treatment alone arm and $10 \%$ in medical treatment plus stent arm reached the end point of $>20 \%$ decline in eGFR ( $p=N S)$. The groups did not differ in BP control or the composite end point of decline in renal function and survival. There were certain inherent problems with the design of this study. The study groups were small to begin with. The follow-up was short and the study had no core laboratory adjudication. Approximately, $28 \%$ of the patients who were randomized to the stent arm did not receive the stent. Medical treatment was poorly defined. The authors of the STAR trial concluded that their results are compatible with both efficacy and harm and therefore were inconclusive.

\section{ASTRAL trial}

Angioplasty and Stenting for Renal Artery Lesions [25] was a multi centric randomized trial conducted in European centers in which 806 patients with at least one RAS $>50 \%$ and whose physicians were uncertain whether early revascularization was clinically indicated were randomized to stenting and medical therapy vs. medical therapy alone. The primary end point which was defined as a change in renal function measured by the reciprocal of the serum creatinine level, was not significantly different between the two study groups at 5 years of follow-up. The secondary outcomes, including decline in $\mathrm{BP}$ and rates of myocardial infarctions, cerebrovascular events, congestive heart failure or risk adjusted mortality were similar in both treatment arms. As was seen with earlier randomized studies, the ASTRAL trial also had certain important limitations. The study included many patients without clinically significant lesions who would not benefit from renal stenting. Also, this trial was underpowered to detect the difference in cardiovascular events. Almost a quarter of patients who were randomized to stent arm underwent angioplasty only without a stent placement. Twelve percent of the patients who received a stent had a residual stenosis $>50 \%$, and $7 \%$ of the patients in the stent arm had angioplasty only. There were high complication rates and the medical treatment was not well defined. There were 3 procedure related deaths reported in this study.

Therefore, 3 of these trials had serious flaws in their design. None of the studies were designed to have an adequate power to evaluate a composite end point of cardiovascular outcomes. All only evaluated the surrogate endpoints. The major issue with these trials was that the patients with clinically significant lesions who would benefit from renal stenting were not included in the studies. The medical management was either imprecisely defined or inadequate in each of the trials. Thus, the best treatment option for the treatment of RAS continues to remain elusive [26].

The Cardiovascular Outcomes in Renal Atherosclerotic Lesions (CORAL) [27] study is a large multi centric, randomized trial which is expected to be completed in 2012. The study includes the patients, with greater than $60 \%$ stenosis and receiving at least two anti-hypertensive medications, and with creatinine $<3 \mathrm{mg} / \mathrm{dL}$. This trial is comparing renal stenting vs. optimal medical management, and is expected to help us understand whether renal stenting improves renal function, patient survival, cardiovascular outcomes and quality of life. There are separate core laboratories which will analyze all angiograms, allocation of medical treatment, laboratory work up and other imaging tests. Pending the results of the CORAL study, the best option for the treatment of RAS remains unclear. Another ongoing trial is looking at the effect of stenting with optimal medical management vs. medical management alone on the renal function at 12 months following stenting. Another study RADAR is currently going on and is looking at the effect of renal artery stenting when compared to medical therapy alone [28].

\section{Current practice and guidelines}

According to the current guidelines on peripheral artery disease [29], revascularization in patients suffering from RAS and recurrent episodes of congestive heart failure have received a class I 
indication. They have recommended renal revascularization as a class IIa indication in patients with (i) global renal ischemia, (ii) progressive chronic kidney disease, (iii) unstable angina (worsening, or resistant), (iv) hypertension that is worsening, resistant, malignant or associated with an unexplained unilateral small kidney or in patients who cannot tolerate antihypertensive medications.

\section{Future direction}

\section{Clinical significance of a hemodynamically significant stenosis}

RAS causes a drop in BP distal to the site of occlusion. In a unilateral stenosis this drop in pressure may act as a stimulus for the activation of renin-angiotensin-aldosterone axis. The contra lateral kidney responds by pressure natreuresis in order to lower BP. This fall in BP further decreases the $\mathrm{BP}$ and perfusion distal to the stenotic lesion in the affected kidney [30]. This drop in BP is important for the development and perpetuation of renovascular hypertension. Thus what constitutes a hemodynamically significant lesion is important for an interventionalist to know because these hemodynamically significant lesions are more likely to respond to the renal angioplasty and stenting. De Bruyne et al. [30] obtained trans-stenotic pressure gradient before and after unilateral stenting in 15 patients. Stenosis severity of was expressed as the ratio of distal pressure $\left(\mathrm{P}_{\mathrm{D}}\right)$ to the aortic pressure $\left(\mathrm{P}_{\mathrm{A}}\right)$. Baloon inflation was adjusted so as to create $60 \%$ of stenosis with a controlled pressure gradient of 1 to 0.5 between the aorta $\left(\mathrm{P}_{\mathrm{A}}\right)$ and the distal part of the renal artery $\left(\mathrm{P}_{\mathrm{D}}\right)$ with each step lasting $10 \mathrm{~min}$. The plasma renin concentrations were measured at the end of each step in the aorta and both renal veins. In this study they found that when the $\mathrm{P}_{\mathrm{D}} / \mathrm{P}_{\mathrm{A}}$ ratio is greater than 0.9 there was no significant change in the levels of renin concentrations observed. Thus, a lesion with a $\mathrm{P}_{\mathrm{D}} / \mathrm{P}_{\mathrm{A}}$ ratio of greater than 0.9 is unlikely to give rise to renovascular hypertension. However, a $\mathrm{P}_{\mathrm{D}} / \mathrm{P}_{\mathrm{A}}$ ratio of less than 0.9 resulted in a significant increase in the renin levels as measured from the renal vein sampling of the stenotic kidney. The renin levels returned to baseline when the stenosis was relieved. Interestingly plasma renin concentrations also increased in the contralateral non stenotic kidneys. Evaluations for hemodynamically significant lesions in patients with renal stenosis may help improve proper patient selection for the angioplasty and stenting. In a recent study Lessar et al. [31] the role of renal translesional pressure gradient and intravascular ultra- sound in predicting the improvement in hypertension following renal artery stenting in patients with RAS was evaluated. In this study 62 patients had translesional pressure gradient, resting and hyperemic systolic gradient (HSG), fractional flow reserve, and mean gradient measured by a guidewire and angiographic parameters including minimum lumen area and diameter, area stenosis, and diameter stenosis measured quantitatively by intravascular ultrasound. $\mathrm{HSG} \geq 21 \mathrm{~mm} \mathrm{Hg}$ was found to be an independent predictor of improvement in hypertension following renal stenting, with decrease in the number of anti hypertensive medication in the group with $\mathrm{HSG} \geq 21 \mathrm{~mm} \mathrm{Hg}$.

\section{Role of distal embolization and embolic protection devices during renal stenting and angioplasty}

Renal stenting has been reported to result in the peri-procedural loss of renal function in some patients [32-37]. There are multiple factors that might be responsible for this reported periprocedural loss of renal function but the most likely being distal embolization. Distal embolization is very common during renal stenting and angioplasty and as such the interest in the protected renal stenting has evolved. Earlier studies on the use of distal protection during renal stenting reported better outcomes. However, the only prospective randomized controlled trial evaluating role of distal protection with and without abciximab in patient undergoing renal stenting (RESIST) [35] failed to show any benefit of an embolic protection alone. There could have been multiple reasons for the failure of embolic protection device in preserving renal function including incomplete protection, incomplete apposition of the device to the vessel wall, embolization prior to deployement of the device and smaller size of the embolized material. Most importantly, RESIST study was underpowered due to its small size to detect any benefit of embolic protection.

\section{Role of antiplatelet therapy during renal artery stenting and angioplasty}

In the RESIST [35] trail, Cooper et al. [35] reports the effects of renal stenting with and without distal protection device as well as with and without abciximab on the percent change in MDRD GFR at 1 month when compared to the baseline. In this $2 \mathrm{X} 2$ factorial designed study almost 100 patients were randomized to either stenting alone, stenting with embolic protection, stenting with abciximab and stenting with both abciximab and embolic protection. The angiographic analysis, the analysis of fil- 
ter contents and the analysis of renal function were performed at 3 separate core laboratories respectively, with analyzers blinded to both the treatment and clinical outcomes. Stenting alone, stenting with embolic protection and stenting with abciximab were associated with decline in renal function at 1 month. However, an unanticipated interaction was observed between abciximab and embolic protection device with improvement in renal function observed in the group randomized to renal stenting with both abciximab and embolic protection.

Further subgroup analysis of the RESIST trial demonstrated that the embolized material captured was predominantly platelet rich suggesting platelet activation is common during renal stenting $[38,39]$.

In an another subgroup analysis on the use of thienopyridines in the RESIST trial it was shown that the thienopyridines significantly reduced embolization of platelet rich emboli [39]. Use of thienopyridines in subgroup of patients who were randomized to both embolic protection and abciximab was associated with no distal embolization. Thus, the use of thienopyridines may be additive or even synergestic when combined with use of abciximab [39]. However thienopyridine use was not associated with improvement in renal function at 1 month despite reducing distal embolization. This might have been possibly because the use of thienopyridine antiplatelet was not randomly allocated rather was clinically prescribed. Further, the study was not powered enough to detect the thienopyridine effect [39].

\section{Controversies in the diagnosis of renal artery stenosis}

The diagnosis of RAS has been as controversial as has been the optimal management of the RAS. Renal angiography is the gold standard for diagnosis of the renal stenosis. However, this procedure is invasive with risks of adverse events including vessel injury and contrast nephropathy. It is crucial for an interventionalist to know the anatomy and status of the renal artery before the patient is taken for angioplasty. The pretest probability of RAS plays an important role in deciding about the choice of diagnostic modality. Although the prevalance of renal stenosis in general hypertensive population is $1-5 \%$, in patients who have a high pretest probability of having RAS, the prevalance may be as high as $40 \%$ in some patients [40, 41]. Besides renal angiography, the other diagnostic tests available for the diagnosis of the RAS include, duplex ultrasonography, computed tomographic angiography (CTA), MRA, captopril scinitigraphy and captopril test. In a meta-analysis by Boudewijn et al. [41] it was found that both CTA and MRA were equally good in the diagnosis of the RAS (both had a area under ROC curve of 0.99). Both, were found to be superior to duplex ultrasound and the captopril scintigraphy $(p=0.02)$. Duplex ultrasonography and captopril scintigraphy were both superior to the captopril test $(p=0.01)$. Renal angiography was used as a gold standard for the diagnosis of RAS in each of the studies included in the meta-analysis. The diagnosis of the RAS can be made with the use of duplex ultrasonography but if the results of the ultrasonography are inconclusive or technically limited, MRA or CTA should be used [28].

\section{Conclusions}

The diagnosis as well as the optimal medical management of the patient suffering from RAS continues to be controversial. In future we expect CORAL and RADAR trials might help answer many questions which earlier trials failed to do.

\section{Conflict of interest: none declared}

\section{References}

1. Hansen KJ, Edwards MS, Craven TE et al. Prevalence of renovascular disease in the elderly: A population-based study. J Vasc Surg, 2002; 36: 443-451.

2. Kalra PA, Guo H, Kausz AT et al. Atherosclerotic renovascular disease in United States patients aged 67 years or older: Risk factors, revascularization, and prognosis. Kidney Int, 2005; 68: 293-301.

3. Hansen KJ, Edwards MS, Craven TE et al. Prevalence of renovascular disease in the elderly: A population-based study. J Vasc Surg, 2002; 36: 443-451.

4. Caps MT, Zierler RE, Polissar NL et al. Risk of atrophy in kidneys with atherosclerotic renal artery stenosis. Kidney Int, 1998; 53: 735-742.

5. Rimmer JM, Gennari FJ. Atherosclerotic renovascular disease and progressive renal failure. Ann Intern Med, 1993; 118: 712-719 .

6. Tegtmeyer CJ, Selby JB, Hartwell GD, Ayers C, Tegtmeyer V. Results and complications of angioplasty in fibromuscular disease. Circulation, 1991; 83 (2 suppl.): I155-I161.

7. van Jaarsveld BC, Krijnen P, Pieterman $\mathrm{H}$ et al. The effect of balloon angioplasty on hypertension in atherosclerotic renal-artery stenosis. Dutch Renal Artery Stenosis Intervention Cooperative Study Group. N Engl J Med, 2000; 342: 1007-1014.

8. Chábová V, Schirger A, Stanson AW, McKusick MA, Textor SC. Outcomes of atherosclerotic renal artery stenosis managed without revascularization. Mayo Clin Proc, 2000; 75: 437-444.

9. Wright JT Jr, Bakris G, Greene T et al.; African American Study of Kidney Disease and Hypertension Study Group. Effect of blood pressure lowering and antihypertensive drug class on progression of hypertensive kidney disease: Results from the AASK trial. JAMA, 2002; 288: 2421-2431. 
10. Suresh M, Laboi P, Mamtora H, Kalra PA. Relationship of renal dysfunction to proximal arterial disease severity in atherosclerotic renovascular disease. Nephrol Dial Transplant, 2000; 15: 631-636.

11. Dorros G, Jaff M, Mathiak L et al. Four-year follow-up of Palmaz-Schatz stent revascularization as treatment for atherosclerotic renal artery stenosis. Circulation, 1998; 98: 642-647.

12. Conlon PJ, Little MA, Pieper K, Mark DB. Severity of renal vascular disease predicts mortality in patients undergoing coronary angiography. Kidney Int, 2001; 60: 1490-1497.

13. Valentine RJ, Clagett GP, Miller GL, Myers SI, Martin JD, Chervu A. The coronary risk of unsuspected renal artery stenosis. J Vasc Surg, 1993; 18: 433-439.

14. Vensel LA, Devereux RB, Pickering TG, Herrold EM, Borer JS, Laragh JH. Cardiac structure and function in renovascular hypertension produced by unilateral and bilateral renal artery stenosis. Am J Cardiol, 1986; 58: 575-582.

15. Meissner MD, Wilson AR, Jessup M. Renal artery stenosis in heart failure. Am J Cardiol, 1988; 62: 1307-1308.

16. Johansson M, Herlitz H, Jensen G, Rundqvist B, Friberg P. Increasedcardiovascular mortality in hypertensive patients with renal artery stenosis. Relation to sympathetic activation, renal function and treatment regimens. J Hypertens, 1999; 17 (12 Part 1): 1743-1750.

17. Kennedy DJ, Burket MW, Khuder SA, Shapiro JI, Topp RV, Cooper CJ. Quality of life improves after renal artery stenting. Biol Res Nurs, 2006; 8: 129-137.

18. Kennedy DJ, Colyer WR, Brewster PS et al. Renal insufficiency as a predictor of adverse events and mortality after renal artery stent placement. Am J Kidney Dis, 2003; 42: 926-935.

19. Leertouwer TC, Gussenhoven EJ, Bosch JL et al. Stent placement for renal arterial stenosis: Where do we stand? A metaanalysis. Radiology, 2000; 216: 78-85.

20. Plouin PF, Chatellier G, Darné B, Raynaud A. Blood pressure outcome of angioplasty in atherosclerotic renal artery stenosis: A randomized trial. Essai Multicentrique Medicaments vs. Angioplastie (EMMA) Study Group. Hypertension, 1998; 31: 823-829.

21. Webster J, Marshall F, Abdalla M et al. Randomised comparison of percutaneous angioplasty vs continued medical therapy for hypertensive patients with atheromatous renal artery stenosis. Scottish and Newcastle Renal Artery Stenosis Collaborative Group. J Hum Hypertens, 1998; 12: 329-335.

22. van Jaarsveld BC, Krijnen P, Pieterman $H$ et al. The effect of balloon angioplasty on hypertension in atherosclerotic renal-artery stenosis. Dutch Renal Artery Stenosis Intervention Cooperative Study Group. N Engl J Med, 2000; 342: 1007-1014.

23. Scarpioni R, Michieletti E, Cristinelli L et al. Atherosclerotic renovascular disease: Medical therapy versus medical therapy plus renal artery stenting in preventing renal failure progression: The rationale and study design of a prospective, multicenter and randomized trial (NITER). J Nephrol, 2005; 18: 423-428.

24. Bax L, Woittiez AJ, Kouwenberg HJ et al. Stent placement in patients with atherosclerotic renal artery stenosis and impaired renal function: A randomized trial. Ann Intern Med, 2009; 150: 840-848 (W150-1).

25. Wheatley K, Ives N, Gray R et al.; ASTRAL Investigators. Revascularization versus medical therapy for renal-artery stenosis. N Engl J Med, 2009; 361: 1953-1962.

26. Dworkin LD, Cooper CJ. Clinical practice. Renal-artery stenosis. N Engl J Med, 2009; 361: 1972-1978.

27. Cooper CJ, Murphy TP, Matsumoto A et al. Stent revascularization for the prevention of cardiovascular and renal events among patients with renal artery stenosis and systolic hypertension: Rationale and design of the CORAL trial. Am Heart J, 2006; 152: 59-66.

28. Schwarzwalder U, Hauk M, Zeller T. RADAR: A randomised, multi-centre, prospective study comparing best medical treatment versus best medical treatment plus renal artery stenting in patients with haemodynamically relevant atherosclerotic renal artery stenosis. Trials, 2009; 10: 60.

29. Hirsch AT, Haskal ZJ, Hertzer NR et al. American Association for Vascular Surgery/Society for Vascular Surgery; Society for Cardiovascular Angiography and Interventions; Society for Vascular Medicine and Biology; Society of Interventional Radiology; ACC/AHA Task Force on Practice Guidelines. ACC/AHA Guidelines for the Management of Patientswith Peripheral Arterial Disease (lower extremity, renal, mesenteric, and abdominal aortic): A collaborative report from the American Associations for Vascular Surgery/Society for Vascular Surgery, Society for Cardiovascular Angiography and Interventions, Society for Vascular Medicine and Biology, Society of Interventional Radiology, and the ACC/AHA Task Force on Practice Guideline (writing committee to develop guidelines for the management of patients with peripheral arterial disease): Summary of recommendations. J Vasc Interv Radiol, 2006; 17: 1383-1397.

30. De Bruyne B, Manoharan G, Pijls NH et al. Assessment of renal artery stenosis severity by pressure gradient measurements. J Am Coll Cardiol. 2006; 48: 1851-1855.

31. Leesar MA, Varma J, Shapira A et al. Prediction of hypertension improvement after stenting of renal artery stenosis: Comparative accuracy of translesional pressure gradients, intravascular ultrasound, and angiography. J Am Coll Cardiol, 2009; 53: 2363$-2371$.

32. Henry M, Henry I, Polydorou A, Hugel M. Embolic protection for renal artery stenting. J Cardiovasc Surg (Torino), 2008; 49: 571-589.

33. Edwards MS, Craven BL, Stafford J et al. Distal embolic protection during renal artery angioplasty and stenting. J Vasc Surg, 2006; 44: 128-135.

34. Holden A, Hill A, Jaff MR, Pilmore H. Renal artery stent revascularization with embolic protection in patients with ischemic nephropathy. Kidney Int, 2006; 70: 948-955.

35. Cooper CJ, Haller ST, Colyer W et al. Embolic protection and platelet inhibition during renal artery stenting. Circulation, 2008; 117: 2752-2760.

36. Kawarada O, Yokoi Y, Takemoto K. The characteristics of dissemination of embolic materials during renal artery stenting. Catheter Cardiovasc Interv, 2007; 70: 784-788.

37. Hiramoto J, Hansen KJ, Pan XM, Edwards MS, Sawhney R, Rapp JH. Atheroemboli during renal artery angioplasty: An ex vivo study. J Vasc Surg, 2005; 41: 1026-1030.

38. Kanjwal K, Haller S, Steffes M et al. Complete versus partial distal embolic protection during renal artery stenting. Catheter Cardiovasc Interv, 2009; 73: 725-730.

39. Kanjwal K, Cooper CJ, Virmani R et al. Predictors of embolization during protected renal artery angioplasty and stenting: Role of antiplatelet therapy. Catheter Cardiovasc Interv, 2010; 76: $16-23$.

40. Working Group on Renovascular Hypertension. Detection, evaluation, and treatment of renovascular hypertension. Final report. Arch Intern Med, 1987; 147: 820-829.

41. Boudewijn G, Vasbinder GB, Nelemans PJ et al. Diagnostic tests for renal artery stenosis in patients suspected of having renovascular hypertension: A meta-analysis. Ann Intern Med, 2001; 135: 401-411. 